\title{
SUPORTE SOCIAL E COMPORTAMENTOS ADITIVOS EM ADOLESCENTES PRÉ-UNIVERSITÁRIOS
}

\author{
M. Arriaga, J. Claudino y R. Cordeiro \\ Escola Superior de Enfermagem de Portalegre, Portugal
}

\section{INTRODUÇÃO}

A fase da adolescência, pelo potencial de mudanças que encerra, tem-se tornado um vasto campo de investigações. É na adolescência que ocorre uma parte importante da reorganização do sentido de identidade, envolvendo os aspectos do desenvolvimento e estabelecimento de relações, tendo sido, por isso, escolhido para objecto do nosso estudo a influência da rede de suporte social (família, grupo de amigos e escola) dos adolescentes na adopção de comportamentos aditivos.

A avaliação destes conceitos em populações adolescentes pode ter um importante valor preditivo no seu desenvolvimento psicossocial.

Enunciaram-se os seguintes objectivos para o estudo em questão:

\section{Objectivo geral}

? Analisar a relação entre a satisfação com o suporte social e a adopção de comportamentos aditivos em adolescentes pré-universitários.

\section{Objectivos específicos}

? Avaliar a satisfação dos adolescentes pré-universitários sobre o suporte social.

? Identificar quais os comportamentos aditivos em adolescentes pré-universitários.

? Identificar qual a relação entre a satisfação com o suporte social do adolescente e a adopção de comportamentos aditivos.

No tratamento dos dados, para além dos dados da estatística descritiva, recorremos como teste de verificação da significância estatística das relações entre variáveis, ao coeficiente de correlação de Spearman $\left(r_{s}\right)$.

Os resultados obtidos confirmam alguns estudos anteriormente realizados, com destaque para o aumento significativo dos valores obtidos no consumo de algumas substâncias ilícitas e das lícitas.

Confirma-se que os valores de consumo de bebidas alcoólicas têm aumentado, quando comparadas com estudos semelhantes anteriores (1999 e 2003), verificando-se um maior consumo das bebidas espirituosas/brancas e de cervejas. Os bares e Pub's são o local de preferência para esses consumos.

Ao nível da satisfação com o Suporte Social os resultados obtidos sugerem-nos que os adolescentes estão satisfeitos com o seu suporte social, sentindo maior satisfação com a amizade e a família. 
No que se refere à relação entre a satisfação com o suporte social e a adopção de comportamentos aditivos, os resultados do estudo apontam para uma relação estatisticamente significativa $(p<0,05)$, embora com resultados de correlação que podem ser considerados fracos $(r<0,5)$, entre as variáveis Consumo de Bebidas Alcoólicas Durante os Últimos 30 Dias $(r=0,174)$, Consumo de Cerveja Durante os Últimos 30 Dias $(r=0,156)$, Local do Último Consumo de Bebidas Alcoólicas $(r=0,167)$, Consumo de 5 ou Mais Bebidas Alcoólicas, de Seguida, Durante os Últimos 30 Dias $(r=0,136)$, Uso de Tranquilizante/Sedativos, Sem Indicação Médica, ao Longo da Vida $(r=-0,238)$.

Tais valores sugerem que a adopção de comportamentos aditivos na adolescência está, de alguma forma, associada à satisfação que cada indivíduo tem com o seu suporte social.

\section{METODOLOGIA}

Uma população de estudo de 370 alunos, com uma média de idades de 17,71 anos, matriculados no $12^{\circ}$ Ano de Escolaridade, no ano lectivo de 2004/2005, em duas Escolas Secundárias da rede do Ministério da Educação, situadas na cidade de Portalegre, foi inquirida através de um Questionário de aplicação directa, tendo respondido um total de 262 alunos (45\% ( $n=118)$ do sexo masculino e 55\% ( $n=144)$ do sexo feminino). O grupo em estudo reside maioritariamente no Concelho de Portalegre (63\%), estando matriculados pela $1^{\underline{a}}$ vez no $12^{\circ}$ Ano, um total de $85,5 \%$ do grupo em estudo.

Num estudo descritivo e transversal foram introduzidos os seguintes instrumentos de colheita de dados: ESPAD (European School Survey on Alcohol and other Drugs) adaptada da escala original e a ESSS (Escala de Satisfação com o Suporte Social). (Ribeiro, 1999).

Foram ainda introduzidas algumas questões de caracterização das variáveis: Sexo, Idade, Concelho de Residência, N. de Anos Matriculado no $12^{\circ}$ Ano, Curso que está a frequentar e dentro deste o curso/agrupamento em que está matriculado.

\section{Escalas de medida}

? O ESPAD - European School Survey on Alcohol and Other Drugs (Inquérito Europeu sobre o Consumo de Álcool e Outras Drogas), é um estudo efectuado a nível europeu, por equipas de investigadores independentes, com a coordenação do CAN - The Swedish Council for Information on Alcohol and Other Drugs, que tem o apoio do Grupo Pompidou do Conselho da Europa e de instituições da maior relevância na área do álcool e das drogas, nos diferentes países participantes. Em Portugal, está a cargo do Observatório da Droga e da Toxicodependência, do Instituto da Droga e da Toxicodependência (IDT). O Observatório Europeu da Droga e da Toxicodependência (OEDT) acompanha este estudo com o estatuto de observador. O estudo realiza-se com a periodicidade de 4 anos, tendo sido desenvolvido em 1995, 1999 e 2003. Portugal participa desde o primeiro ano. Do ESPAD original foram introduzidas apenas as seguintes questões: 
$\approx$ Consumo de Tabaco ao Longo da Vida

$\checkmark$ Consumo de Tabaco nos Últimos 30 Dias

2SConsumo de Bebidas Alcoólicas ao Longo da Vida

2. Consumo de Bebidas Alcoólicas durante os Últimos 30 Dias

2Consumo de Cerveja durante os Últimos 30 Dias

\&Consumo de Vinho durante os Últimos 30 Dias

\&. Consumo de Bebidas Espirituosas/Destiladas durante os Últimos 30 Dias

eConsumo de 5 ou mais Bebidas Alcoólicas, de Seguida, durante os Últimos 30 Dias

Estado de Embriaguês nos Últimos 30 Dias

Uso de Tranquilizantes ou Sedativos, sem Indicação Médica, ao Longo da Vida

EUso de Anfetaminas ao Longo da Vida

EUso de LSD ou Outros Alucinogénios ao Longo da Vida

¿ Uso de Crack ao Longo da Vida

\&uso de Cocaína ao Longo da Vida

\&uso de Heroína ao Longo da Vida

Uso de Ecstasy ao Longo da Vida

Uso de "Cogumelos Mágicos" ao Longo da Vida

ex Uso de Esteróides Anabolizantes ao Longo da Vida

? A Escala de Satisfação com o Suporte Social (a seguir designada por ESSS) (Ribeiro, 1999), que mede a satisfação com o suporte social.

A ESSS é constituída por 15 frases que são apresentadas para auto-preenchimento, como um conjunto de afirmações. O sujeito deve assinalar o grau em que concorda com a afirmação (se ela se aplica a ele), numa escala de Likert com cinco posições, "concordo totalmente", "concordo na maior parte", "não concordo nem discordo", "discordo a maior parte" e "discordo totalmente". (Ribeiro, 1999)

Estas 15 frases/itens distribuem-se por quatro (4) dimensões ou factores geradas empiricamente para medir os seguintes aspectos do Suporte Social:

es- o primeiro factor, baptizado "Satisfação com a Amizade", mede a satisfação com as amizades/amigos que o adolescente tem. Inclui cinco (5) itens que correspondem ás proposições 3, 12, 13, 14 e 15 e têm, no estudo de Ribeiro (1999) uma consistência interna, medida pelo Alpha de Cronbach de $?=0,83$ tendo no estudo actual um $?=0,80$;

\&- 0 segundo factor, baptizado "Intimidade" (IN), mede a percepção da existência de suporte social intimo. Inclui quatro itens (itens $1,4,5$ e 6 ) que têm a consistência interna de $?=0,74$ (Ribeiro, 1999) tendo no estudo actual um ? =0,49; 
e- o terceiro factor, baptizado "Satisfação com a Família", mede a satisfação do adolescente com o suporte social familiar existente. Inclui três (3) itens que correspondem às proposições 9, 10 e 11, e têm uma consistência interna de ?=0,74 (Ribeiro, 1999) tendo no estudo actual um ?=0,83;

\&- o último factor gerado, baptizado "Actividades Sociais", mede a satisfação com as actividades sociais que 0 adolescente realiza. Inclui três itens que equivalem às proposições 2, 7 e 8 que têm uma consistência interna de ?=0,64 (Ribeiro, 1999) tendo no estudo actual um $?=0,48$.

A escala total original (Ribeiro, 1999) mostra uma consistência interna de $?=0,85$ tendo no estudo actual um $?=0,80$.

A nota final da escala resulta da soma da totalidade dos itens. A nota de cada dimensão resulta da soma dos itens de cada dimensão ou sub-escala.

Os itens são cotados atribuindo o valor "1" aos itens assinalados em "A", e "5" aos assinalados em "E". São excepção os itens invertidos que são os seguintes: 4,5,9,10,11,12,13,14 e15.

A nota para a escala total pode variar entre 15 e 75 e à nota mais alta corresponde uma percepção de maior suporte social.

\section{RESULTADOS}

\section{Estudo dos Comportamentos Aditivos}

É importante referir que ao longo da apresentação dos resultados, os mesmos irão ser confrontados com os resultados obtidos noutros estudos de investigação, nomeadamente o European School Survey on Alcohol and other Drugs - ESPAD 99 e o European School Survey on Alcohol and other Drugs - ESPAD 03.

Os estudos foram realizados a nível Europeu, tendo participado no primeiro cerca de 30 países e no segundo cerca de 35 países. Na nossa discussão vamos utilizar apenas os dados referentes a Portugal do estudo "ESPAD 99" e "ESPAD 03".

O "ESPAD 99" teve como população alvo os alunos nascidos em 1983, que estavam matriculados no $8 \% / 9 \% 10^{\circ}$ Anos do ensino oficial, e que representavam cerca de $83 \%$ dos estudantes desta idade matriculados no ensino oficial. As escolas e turmas foram escolhidas aleatoriamente, onde foram apurados 3609 estudantes (530 alunos do 8ํㅡo, 879 alunos do 9 Ano e 2200 alunos do 10ํAno).

A dimensão da amostra do "ESPAD 03" foi de cerca de 18000 alunos do $7^{\circ}$ ao $12^{\circ}$ Anos, assegurando a representatividade a nível de Portugal Continental, para cada Ano e grupo de escolaridade bem como para grupo etário dos 13 aos 18 Anos. 
Quadro 1

Resultados comparativos do ESPAD 99, ESPAD 03 e do Estudo Actual 05

\begin{tabular}{|c|c|c|c|}
\hline Variáveis & ESPAD 99 & ESPAD 03 & $\begin{array}{l}\text { Estudo Actual } \\
05\end{array}$ \\
\hline $\begin{array}{l}\text { Consumo de Tabaco ao Longo da } \\
\text { Vida }\end{array}$ & $59,0 \%$ & $62,5 \%$ & $77,8 \%$ \\
\hline $\begin{array}{l}\text { Consumo de Tabaco nos Últimos } 30 \\
\text { Dias }\end{array}$ & $30,8 \%$ & $27,6 \%$ & $53,0 \%$ \\
\hline $\begin{array}{l}\text { Consumo de Bebidas Alcoólicas ao } \\
\text { Longo da Vida }\end{array}$ & $78,1 \%$ & $78,2 \%$ & $92,7 \%$ \\
\hline $\begin{array}{l}\text { Consumo de Bebidas Alcoólicas } \\
\text { durante os Últimos } 30 \text { Dias }\end{array}$ & $48,3 \%$ & $48,7 \%$ & $85,1 \%$ \\
\hline $\begin{array}{l}\text { Consumo de Cerveja durante os } \\
\text { Últimos } 30 \text { Dias }\end{array}$ & $36,9 \%$ & $35,2 \%$ & $64,1 \%$ \\
\hline $\begin{array}{l}\text { Consumo de Vinho durante os } \\
\text { Últimos } 30 \text { Dias }\end{array}$ & $15,2 \%$ & $14,8 \%$ & $56,1 \%$ \\
\hline $\begin{array}{l}\text { Consumo de } \quad \text { Bebidas } \\
\text { Espirituosas/Destiladas durante os }\end{array}$ & $44,1 \%$ & $51,4 \%$ & $80.1 \%$ \\
\hline $\begin{array}{l}\text { Últimos } 30 \text { Dias } \\
\text { Consumo de } 5 \text { ou mais Bebidas }\end{array}$ & & & \\
\hline $\begin{array}{l}\text { Consumo de } 5 \text { ou mais Bebidas } \\
\text { Alcoólicas, de Seguida, durante os } \\
\text { Últimos } 30 \text { Dias }\end{array}$ & $23,2 \%$ & $25,5 \%$ & $65,3 \%$ \\
\hline $\begin{array}{l}\text { Estado de Embriaguês nos Últimos } 30 \\
\text { Dias } \\
\text { Uso de Tranquilizantes ou Sedativos, } \\
\text { sem Indicação Médica, ao Longo da } \\
\text { Vida }\end{array}$ & $14,2 \%$ & $14,5 \%$ & $47,3 \%$ \\
\hline $\begin{array}{l}\text { Vida } \\
\text { Uso de Anfetaminas ao Longo da } \\
\text { Vida }\end{array}$ & $7,6 \%$ & $5,2 \%$ & $13,0^{\circ}$ \\
\hline $\begin{array}{l}\text { Uso de LSD ou Outros Alucinogéneos } \\
\text { ao Longo da Vida }\end{array}$ & $1,0 \%$ & $2,1 \%$ & $3,1 \%$ \\
\hline $\begin{array}{l}\text { Uso de Crack ao Longo da Vida } \\
\text { Uso de Cocaína ao Longo da Vida } \\
\text { Uso de Heroína ao Longo da Vida } \\
\text { Uso de Ecstasy ao Longo da Vida }\end{array}$ & $\begin{array}{l}0,8 \% \\
0,8 \% \\
2,5 \% \\
2,3 \%\end{array}$ & $\begin{array}{l}1,6 \% \\
2,6 \% \\
1,8 \% \\
4,0 \%\end{array}$ & $\begin{array}{l}0,4 \% \\
1,9 \% \\
0,8 \% \\
3,1 \%\end{array}$ \\
\hline $\begin{array}{l}\text { Uso de "Cogumelos Mágicos" ao } \\
\text { Longo da Vida }\end{array}$ & $1,0 \%$ & $3,0 \%$ & $5,7 \%$ \\
\hline $\begin{array}{l}\text { Uso de Esteróides Anabolizantes ao } \\
\text { Longo da Vida }\end{array}$ & $0,0 \%$ & $1,1 \%$ & $0,0 \%$ \\
\hline
\end{tabular}

\section{Estudo da Satisfação com o Suporte Social}

Quadro 2

Resultados médios e desvios-padrão do Suporte Social e Dimensões

\begin{tabular}{|l|r|r|r|}
\hline & \multicolumn{1}{|c|}{$\mathrm{n}$} & \multicolumn{1}{c|}{ Média } & Desvio-padrão \\
\hline Suporte Social & $\mathbf{2 5 7}$ & $\mathbf{3 , 7 9}$ &, 60 \\
Satisfação com a & 259 & $\mathbf{4 , 1 2}$ &, 73 \\
Amizade & 260 & 3,78 &, 79 \\
Intimidade & 261 & 4,01 &, 93 \\
Satisfação com a Família & 261 & 3,27 &, 96 \\
Actividades Sociais & 257 & & \\
Total & & \\
\hline
\end{tabular}




\section{Relações entre Variáveis}

Quadro 3

Correlação entre as Dimensões do Suporte Social e as Variáveis de Caracterização

\begin{tabular}{|c|c|c|c|c|c|}
\hline & $\begin{array}{l}\text { Satisfação } \\
\text { com } \\
\text { Amizade }\end{array}$ & Intimidade & $\begin{array}{l}\text { Satisfação com } \\
\text { a Família }\end{array}$ & $\begin{array}{l}\text { Actividades } \\
\text { Sociais }\end{array}$ & Suporte Social \\
\hline Idade & $\begin{array}{l}r=-0,013 \\
p>0,05\end{array}$ & $\begin{array}{l}r=-0,127^{*} \\
p<0,05\end{array}$ & $\begin{array}{l}r=0,035 \\
p>0,05\end{array}$ & $\begin{array}{l}r=-0,018 \\
p>0,05\end{array}$ & $\begin{array}{l}r=-0,049 \\
p>0,05\end{array}$ \\
\hline Sexo & $\begin{array}{l}r=-0,152^{*} \\
p<0,05\end{array}$ & $\begin{array}{l}r=-0,037 \\
p>0,05\end{array}$ & $\begin{array}{l}r=-0,012 \\
p>0,05\end{array}$ & $\begin{array}{l}r=-0,232^{* *} \\
p<0,01\end{array}$ & $\begin{array}{l}r=-0,147^{*} \\
p<0,05\end{array}$ \\
\hline $\begin{array}{l}\text { Concelho de } \\
\text { Residência }\end{array}$ & $\begin{array}{l}r=0,125^{*} \\
p<0,05\end{array}$ & $\begin{array}{l}r=0,087 \\
p>0,05\end{array}$ & $\begin{array}{l}r=0,027 \\
p>0,05\end{array}$ & $\begin{array}{l}r=0,089 \\
p>0,05\end{array}$ & $\begin{array}{l}r=0,123 \\
p>0,05\end{array}$ \\
\hline $\begin{array}{l}\text { Curso que } \\
\text { Frequenta } \\
\text { № de Anos } \\
\text { Matriculado no } \\
12^{\circ} \text { Ano }\end{array}$ & $\begin{array}{l}r=-0,054 \\
p>0,05 \\
r=-0,073 \\
p>0,05\end{array}$ & $\begin{array}{l}r=-0,099 \\
p>0,05 \\
r=-0,102 \\
p>0,05\end{array}$ & $\begin{array}{l}r=-0,061 \\
p>0,05 \\
r=-0,039 \\
p>0,05\end{array}$ & $\begin{array}{l}r=-0,020 \\
p>0,05 \\
r=-0,072 \\
p>0,05\end{array}$ & $\begin{array}{l}r=-0,094 \\
p>0,05 \\
r=-0,105 \\
p>0,05\end{array}$ \\
\hline
\end{tabular}

* Correlação estatisticamente significativa

** Correlação especialmente significativa

Quadro 4

Correlação entre as Variáveis de Caracterização e as Variáveis relativas Consumo de Tabaco

\begin{tabular}{|c|c|c|}
\hline & $\begin{array}{l}\text { Consumo de } \\
\text { Tabaco ao } \\
\text { Longo da Vida }\end{array}$ & $\begin{array}{l}\text { Consumo de } \\
\text { Tabaco nos } \\
\text { Últimos } 30 \text { dias }\end{array}$ \\
\hline Idade & $\begin{array}{l}r=0,013 \\
p>0,05\end{array}$ & $\begin{array}{l}r=0,132^{*} \\
p<0,05\end{array}$ \\
\hline $\begin{array}{l}\text { Sexo } \\
\text { Concelho de Residência } \\
\text { № de Anos Matriculado no 12\% } \\
\text { Ano } \\
\text { Curso que Frequenta }\end{array}$ & $\begin{array}{l}r=0,280 \\
p>0,05 \\
r=0,450 \\
p>0,05 \\
r=0,310 \\
p>0,05 \\
r=-0,110 \\
p>0,05\end{array}$ & $\begin{array}{l}r=0,019 \\
p>0,05 \\
r=0,121 \\
p>0,05 \\
r=0,075 \\
p>0,05 \\
r=-0,036 \\
p>0,05\end{array}$ \\
\hline
\end{tabular}

* Correlação estatisticamente significativa

Quadro 5

Correlação entre as Variáveis de Caracterização e as Variáveis relativas ao Consumo de Bebidas Alcoólicas

\begin{tabular}{|c|c|c|c|c|c|}
\hline & Idade & Sexo & $\begin{array}{l}\text { Concelho de } \\
\text { Residência }\end{array}$ & $\begin{array}{l}\text { № de Anos } \\
\text { Matriculado no } \\
12^{\circ} \text { Ano }\end{array}$ & $\begin{array}{l}\text { Curso que } \\
\text { Frequenta }\end{array}$ \\
\hline $\begin{array}{l}\text { Consumo de Bebidas } \\
\text { Alcoólicas ao Longo da } \\
\text { Vida }\end{array}$ & $\begin{array}{l}r=-0,072 \\
p>0,05\end{array}$ & $\begin{array}{l}r=-0,294^{* *} \\
p<0,01\end{array}$ & $\begin{array}{l}r=0,08 \\
p>0,05\end{array}$ & $\begin{array}{l}r=-0,007 \\
p>0,05\end{array}$ & $\begin{array}{l}r=-0,180^{* *} \\
p<0,01\end{array}$ \\
\hline $\begin{array}{l}\text { Consumo de Bebidas } \\
\text { Alcoólicas Durante os }\end{array}$ & $\begin{array}{l}r=-0,057 \\
p>0,05\end{array}$ & $\begin{array}{l}r=-0,346^{* *} \\
p<0,01\end{array}$ & $\begin{array}{l}r=0,013 \\
p>0,05\end{array}$ & $\begin{array}{l}r=0,019 \\
p>0,05\end{array}$ & $\begin{array}{l}r=-0,105 \\
p>0,05\end{array}$ \\
\hline $\begin{array}{l}\text { Consumo de Bebidas } \\
\text { Alcoólicas Durante os } \\
\text { Últimos } 30 \text { Dias }\end{array}$ & $\begin{array}{l}r=-0,016 \\
p>0,05\end{array}$ & $\begin{array}{l}r=-0,308^{* *} \\
p<0,01\end{array}$ & $\begin{array}{l}r=-0,025 \\
p>0,05\end{array}$ & $\begin{array}{l}r=0,034 \\
p>0,05\end{array}$ & $\begin{array}{l}r=-0,083 \\
p>0,05\end{array}$ \\
\hline $\begin{array}{l}\text { Auto-Previsão de } \\
\text { Consumo de Bebidas } \\
\text { Alcoólicas aos } 25 \text { Anos }\end{array}$ & $\begin{array}{l}r=0,130^{*} \\
p<0,05\end{array}$ & $\begin{array}{l}r=0,09 \\
p>0,05\end{array}$ & $\begin{array}{l}r=0,006 \\
p>0,05\end{array}$ & $\begin{array}{l}r=0,109 \\
p>0,05\end{array}$ & $\begin{array}{l}r=0,041 \\
p>0,05\end{array}$ \\
\hline $\begin{array}{l}\text { Consumo de Cerveja } \\
\text { Durante os Últimos } 30 \\
\text { Dias }\end{array}$ & $\begin{array}{l}r=-0,013 \\
p>0,05\end{array}$ & $\begin{array}{l}r=-0,471^{* *} \\
p<0,01\end{array}$ & $\begin{array}{l}r=-0,042 \\
p>0,05\end{array}$ & $\begin{array}{l}r=0,033 \\
p>0,05\end{array}$ & $\begin{array}{l}r=-0,018 \\
p>0,05\end{array}$ \\
\hline $\begin{array}{l}\text { Consumo de Vinho } \\
\text { Durante os Últimos } 30 \\
\text { Dias }\end{array}$ & $\begin{array}{l}r=0,099 \\
p>0,05\end{array}$ & $\begin{array}{l}r=-0,265^{* *} \\
p<0,01\end{array}$ & $\begin{array}{l}r=0,020 \\
p>0,05\end{array}$ & $\begin{array}{l}r=0,049 \\
p>0,05\end{array}$ & $\begin{array}{l}r=-0,040 \\
p>0,05\end{array}$ \\
\hline
\end{tabular}




\begin{tabular}{|c|c|c|c|c|c|}
\hline $\begin{array}{l}\text { Consumo de Bebidas } \\
\text { Espirituosas / Destiladas } \\
\text { Durante os Últimos } 30 \\
\text { Dias }\end{array}$ & $\begin{array}{l}r=-0,144^{*} \\
p<0,05\end{array}$ & $\begin{array}{l}r=-0,202^{* *} \\
p<0,01\end{array}$ & $\begin{array}{l}r=-0,062 \\
p>0,05\end{array}$ & $\begin{array}{l}r=-0,002 \\
p>0,05\end{array}$ & $\begin{array}{l}r=-0,158^{*} \\
p<0,05\end{array}$ \\
\hline $\begin{array}{l}\text { Consumo de } 5 \text { ou mais } \\
\text { Bebidas Alcoólicas } \\
\text { Durante os Últimos } 30 \\
\text { Dias }\end{array}$ & $\begin{array}{l}r=-0,056 \\
p>0,05\end{array}$ & $\begin{array}{l}r=-0,348^{* *} \\
p<0,01\end{array}$ & $\begin{array}{l}r=-0,072 \\
p>0,05\end{array}$ & $\begin{array}{l}r=-0,015 \\
p>0,05\end{array}$ & $\begin{array}{l}r=-0,047 \\
p>0,05\end{array}$ \\
\hline $\begin{array}{l}\text { Estado de Embriaguês } \\
\text { em Toda a Vida }\end{array}$ & $\begin{array}{l}r=0,078 \\
p>0,05\end{array}$ & $\begin{array}{l}r=-0,259^{* *} \\
p<0,01\end{array}$ & $\begin{array}{l}r=-0,110 \\
p>0,05\end{array}$ & $\begin{array}{l}r=0,067 \\
p>0,05\end{array}$ & $\begin{array}{l}r=-0,064 \\
p>0,05\end{array}$ \\
\hline $\begin{array}{l}\text { Estado de Embriaguês } \\
\text { nos Últimos } 12 \text { Meses }\end{array}$ & $\begin{array}{l}r=0,032 \\
p>0,05\end{array}$ & $\begin{array}{l}r=-0,254^{* *} \\
p<0,01\end{array}$ & $\begin{array}{l}r=-0,076 \\
p>0,05\end{array}$ & $\begin{array}{l}r=-0,049 \\
p>0,05\end{array}$ & $\begin{array}{l}r=-0,021 \\
p>0,05\end{array}$ \\
\hline $\begin{array}{l}\text { Estado de Embriaguês } \\
\text { nos Últimos } 30 \text { Dias } \\
\text { Gravidade do Ultimo } \\
\text { Estado de Embriaguês }\end{array}$ & $\begin{array}{l}r=0,044 \\
p>0,05 \\
r=0,036 \\
p>0,05\end{array}$ & $\begin{array}{l}r=-0,175^{* *} \\
p<0,01 \\
r=-0,162^{*} \\
p<0,05\end{array}$ & $\begin{array}{l}r=-0,113 \\
p>0,05 \\
r=-0,007 \\
p>0,05\end{array}$ & $\begin{array}{l}r=-0,040 \\
p>0,05 \\
r=-0,030 \\
p>0,05\end{array}$ & $\begin{array}{l}r=-0,015 \\
p>0,05 \\
r=-0,060 \\
p>0,05\end{array}$ \\
\hline
\end{tabular}

* Correlação estatisticamente significativa

** Correlação especialmente significativa

Quadro 6

Correlação entre as Variáveis de Caracterização e as Variáveis relativas às Drogas

\begin{tabular}{|c|c|c|c|c|c|}
\hline & Idade & Sexo & $\begin{array}{l}\text { Concelho de } \\
\text { Residência }\end{array}$ & $\begin{array}{l}\text { № de Anos } \\
\text { Matriculado no } \\
12^{\circ} \text { Ano }\end{array}$ & $\begin{array}{l}\text { Curso que } \\
\text { Frequenta }\end{array}$ \\
\hline $\begin{array}{l}\text { Contacto } \\
\text { Informação }\end{array}$ & $\begin{array}{l}r=0,029 \\
p>0,05\end{array}$ & $\begin{array}{l}r=-0,197^{* *} \\
p<0,01\end{array}$ & $\begin{array}{l}r=-0,040 \\
p>0,05\end{array}$ & $\begin{array}{l}r=0,007 \\
p>0,05\end{array}$ & $\begin{array}{l}r=0,042 \\
p>0,05\end{array}$ \\
\hline $\begin{array}{l}\text { Tranquilizantes ou } \\
\text { Sedativos }\end{array}$ & & & & & \\
\hline $\begin{array}{l}\text { Contacto com } \\
\text { Informação LSD }\end{array}$ & $\begin{array}{l}r=0,175^{* *} \\
p<0,01\end{array}$ & $\begin{array}{l}r=0,111 \\
p>0,05\end{array}$ & $\begin{array}{l}r=-0,018 \\
p>0,05\end{array}$ & $\begin{array}{l}r=0,055 \\
p>0,05\end{array}$ & $\begin{array}{l}r=0,153^{*} \\
p<0,05\end{array}$ \\
\hline $\begin{array}{l}\text { Contacto com } \\
\text { Informação Crack }\end{array}$ & $\begin{array}{l}r=-0,049 \\
p>0,05\end{array}$ & $\begin{array}{l}r=0,213^{* *} \\
p<0,01\end{array}$ & $\begin{array}{l}r=-0,040 \\
p>0,05\end{array}$ & $\begin{array}{l}r=-0,132 * \\
p<0,05\end{array}$ & $\begin{array}{l}r=0,108 \\
p>0,05\end{array}$ \\
\hline $\begin{array}{l}\text { Uso de } \\
\text { Tranquilizantes ou } \\
\text { Sedativos, s/ Ind. } \\
\text { Méd., ao Longo da } \\
\text { Vida }\end{array}$ & $\begin{array}{l}r=0,124^{*} \\
p<0,05\end{array}$ & $\begin{array}{l}r=0,079 \\
p>0,05\end{array}$ & $\begin{array}{l}r=-0,011 \\
p>0,05\end{array}$ & $\begin{array}{l}r=0,228^{* *} \\
p<0,01\end{array}$ & $\begin{array}{l}r=0,064 \\
p>0,05\end{array}$ \\
\hline $\begin{array}{l}\text { Uso de Haxixe ou } \\
\text { Marijuana ao Longo } \\
\text { da Vida }\end{array}$ & $\begin{array}{l}r=0,114 \\
p>0,05\end{array}$ & $\begin{array}{l}r=-0,206^{* *} \\
p<0,01\end{array}$ & $\begin{array}{l}r=-0,047 \\
p>0,05\end{array}$ & $\begin{array}{l}r=0,089 \\
p>0,05\end{array}$ & $\begin{array}{l}r=-0,053 \\
p>0,05\end{array}$ \\
\hline $\begin{array}{l}\text { Uso de Cogumelos } \\
\text { Mágicos, ao Longo } \\
\text { da Vida }\end{array}$ & $\begin{array}{l}r=0,161^{* *} \\
p<0,01\end{array}$ & $\begin{array}{l}r=-0,008 \\
p>0,05\end{array}$ & $\begin{array}{l}r=0,066 \\
p>0,05\end{array}$ & $\begin{array}{l}r=-0,007 \\
p>0,05\end{array}$ & $\begin{array}{l}r=0,090 \\
p>0,05\end{array}$ \\
\hline $\begin{array}{l}\text { Uso de Álcool com } \\
\text { Comprimidos, ao } \\
\text { Longo da Vida }\end{array}$ & $\begin{array}{l}r=0,196^{* *} \\
p<0,01\end{array}$ & $\begin{array}{l}r=-0,085 \\
p>0,05\end{array}$ & $\begin{array}{l}r=0,087 \\
p>0,05\end{array}$ & $\begin{array}{l}r=0,288^{* *} \\
p<0,01\end{array}$ & $\begin{array}{l}r=0,048 \\
p>0,05\end{array}$ \\
\hline
\end{tabular}

${ }^{*}$ Correlação estatisticamente significativa

** Correlação especialmente significativa

\section{QUADRO 7}

Correlação entre as Dimensões do Suporte Social as Variáveis relacionadas com o Consumo de Bebidas Alcoólicas e Consumo de Tabaco

\begin{tabular}{|c|c|c|c|c|c|}
\hline & $\begin{array}{l}\text { Satisfação } \\
\text { com a } \\
\text { Amizade }\end{array}$ & Intimidade & $\begin{array}{l}\text { Satisfação com } \\
\text { a Família }\end{array}$ & $\begin{array}{l}\text { Actividades } \\
\text { Sociais }\end{array}$ & $\begin{array}{l}\text { Suporte } \\
\text { Social }\end{array}$ \\
\hline $\begin{array}{l}\text { Consumo de Tabaco ao Longo } \\
\text { da Vida } \\
\text { Consumo de Tabaco nos } \\
\text { Últimos } 30 \text { Dias } \\
\text { Consumo de Bebidas } \\
\text { Alcoólicas ao Longo da Vida } \\
\text { Consumo de Bebidas } \\
\text { Alcoólicas Durante os Últimos } \\
12 \text { Meses }\end{array}$ & $\begin{array}{l}r=0,009 \\
p>0,05 \\
r=0,021 \\
p>0,05 \\
r=0,015 \\
p>0,05 \\
r=0,066 \\
p>0,05\end{array}$ & $\begin{array}{l}r=-0,016 \\
p>0,05 \\
r=-0,064 \\
p>0,05 \\
r=0,079 \\
p>0,05 \\
r=0,102 \\
p>0,05\end{array}$ & $\begin{array}{l}r=-0,140^{*} \\
p<0,05 \\
r=-0,145^{*} \\
p<0,05 \\
r=-0,152^{*} \\
p<0,05 \\
r=-0,094 \\
p>0,05\end{array}$ & $\begin{array}{l}r=0,116 \\
p>0,05 \\
r=0,058 \\
p>0,05 \\
r=0,209^{* *} \\
p<0,01 \\
r=0,191^{* *} \\
p<0,01\end{array}$ & $\begin{array}{l}r=-0,005 \\
p>0,05 \\
r=-0,044 \\
p>0,05 \\
r=0,063 \\
p>0,05 \\
r=0,115 \\
p>0,05\end{array}$ \\
\hline $\begin{array}{l}\text { Consumo de Bebidas } \\
\text { Alcoólicas Durante os Últimos } \\
30 \text { Dias }\end{array}$ & $\begin{array}{l}r=0,158^{*} \\
p<0,05\end{array}$ & $\begin{array}{l}r=0,167^{* *} \\
p<0,01\end{array}$ & $\begin{array}{l}r=-0,065 \\
p>0,05\end{array}$ & $\begin{array}{l}r=0,212^{* *} \\
p<0,01\end{array}$ & $\begin{array}{l}r=0,174^{* *} \\
p<0,01\end{array}$ \\
\hline
\end{tabular}




\begin{tabular}{|c|c|c|c|c|c|}
\hline $\begin{array}{l}\text { Auto-Previsão de Consumo de } \\
\text { Bebidas Alcoólicas aos } 25 \text { Anos }\end{array}$ & $\begin{array}{l}r=0,100 \\
p>0,05\end{array}$ & $\begin{array}{l}r=-0,054 \\
p>0,05\end{array}$ & $\begin{array}{l}r=0,037 \\
p>0,05\end{array}$ & $\begin{array}{l}r=0,012 \\
p>0,05\end{array}$ & $\begin{array}{l}r=0,052 \\
p>0,05\end{array}$ \\
\hline $\begin{array}{l}\text { Consumo de Cerveja Durante } \\
\text { os Últimos } 30 \text { Dias }\end{array}$ & $\begin{array}{l}r=0,166^{* *} \\
p<0,01\end{array}$ & $\begin{array}{l}r=0,132^{*} \\
p<0,05\end{array}$ & $\begin{array}{l}r=-0,079 \\
p>0,05\end{array}$ & $\begin{array}{l}r=0,205^{* *} \\
p<0,01\end{array}$ & $\begin{array}{l}r=0,156^{*} \\
p<0,05\end{array}$ \\
\hline $\begin{array}{l}\text { Consumo de Vinho Durante os } \\
\text { Últimos } 30 \text { Dias }\end{array}$ & $\begin{array}{l}r=0,109 \\
p>0,05\end{array}$ & $\begin{array}{l}r=0,052 \\
p>0,05\end{array}$ & $\begin{array}{l}r=-0,126^{*} \\
p<0,05\end{array}$ & $\begin{array}{l}r=0,128^{*} \\
p<0,05\end{array}$ & $\begin{array}{l}r=0,063 \\
p>0,05\end{array}$ \\
\hline $\begin{array}{l}\text { Consumo de Bebidas } \\
\text { Espirituosas/Destiladas Durante } \\
\text { os Últimos } 30 \text { Dias }\end{array}$ & $\begin{array}{l}r=0,138^{*} \\
p<0,05\end{array}$ & $\begin{array}{l}r=0,056 \\
p>0,05\end{array}$ & $\begin{array}{l}r=-0,090 \\
p>0,05\end{array}$ & $\begin{array}{l}r=0,040 \\
p>0,05\end{array}$ & $\begin{array}{l}r=0,051 \\
p>0,05\end{array}$ \\
\hline $\begin{array}{l}\text { Local do Último Consumo de } \\
\text { Bebidas Alcoólicas }\end{array}$ & $\begin{array}{l}r=0,120 \\
p>0,05\end{array}$ & $\begin{array}{l}r=0,109 \\
p>0,05\end{array}$ & $\begin{array}{l}r=0,089 \\
p>0,05\end{array}$ & $\begin{array}{l}r=0,107 \\
p>0,05\end{array}$ & $\begin{array}{l}r=0,167^{* *} \\
p<0,01\end{array}$ \\
\hline $\begin{array}{l}\text { Consumo de } 5 \text { ou mais Bebidas } \\
\text { Alcoólicas de Seguida Durante } \\
\text { os Últimos } 30 \text { Dias }\end{array}$ & $\begin{array}{l}r=0,132 * \\
p<0,05\end{array}$ & $\begin{array}{l}r=0,148^{*} \\
p<0,05\end{array}$ & $\begin{array}{l}r=-0,097 \\
p>0,05\end{array}$ & $\begin{array}{l}r=0,165^{* *} \\
p<0,01\end{array}$ & $\begin{array}{l}r=0,136^{*} \\
p<0,05\end{array}$ \\
\hline $\begin{array}{l}\text { Estado de Embriaguês em Toda } \\
\text { a Vida } \\
\text { Estado de Embriaguês nos } \\
\text { Últimos } 12 \text { Meses }\end{array}$ & $\begin{array}{l}r=0,056 \\
p>0,05 \\
r=0,028 \\
p>0,05\end{array}$ & $\begin{array}{l}r=0,012 \\
p>0,05 \\
r=0,063 \\
p>0,05\end{array}$ & $\begin{array}{l}r=-0,105 \\
p>0,05 \\
r=-0,127^{*} \\
p<0,05\end{array}$ & $\begin{array}{l}r=0,156^{*} \\
p<0,05 \\
r=0,132^{*} \\
p<0,05\end{array}$ & $\begin{array}{l}r=0,047 \\
p>0,05 \\
r=0,046 \\
p>0,05\end{array}$ \\
\hline $\begin{array}{l}\text { Estado de Embriaguês nos } \\
\text { Últimos } 30 \text { Dias } \\
\text { Gravidade do Ultimo Estado de } \\
\text { Embriaguês }\end{array}$ & $\begin{array}{l}r=0,054 \\
p>0,05 \\
r=0,089 \\
p>0,05\end{array}$ & $\begin{array}{l}r=0,053 \\
p>0,05 \\
r=0,061 \\
p>0,05\end{array}$ & $\begin{array}{l}r=-0,127^{*} \\
p<0,05 \\
r=-0,023 \\
p>0,05\end{array}$ & $\begin{array}{l}r=0,084 \\
p>0,05 \\
r=0,105 \\
p>0,05\end{array}$ & $\begin{array}{l}r=0,022 \\
p>0,05 \\
r=0,085 \\
p>0,05\end{array}$ \\
\hline
\end{tabular}

* Correlação estatisticamente significativa

** Correlação especialmente significativa

Quadro 8

Correlação entre as Dimensões do Suporte Social as Variáveis relacionadas com o Contacto com Informação ou Uso de Drogas

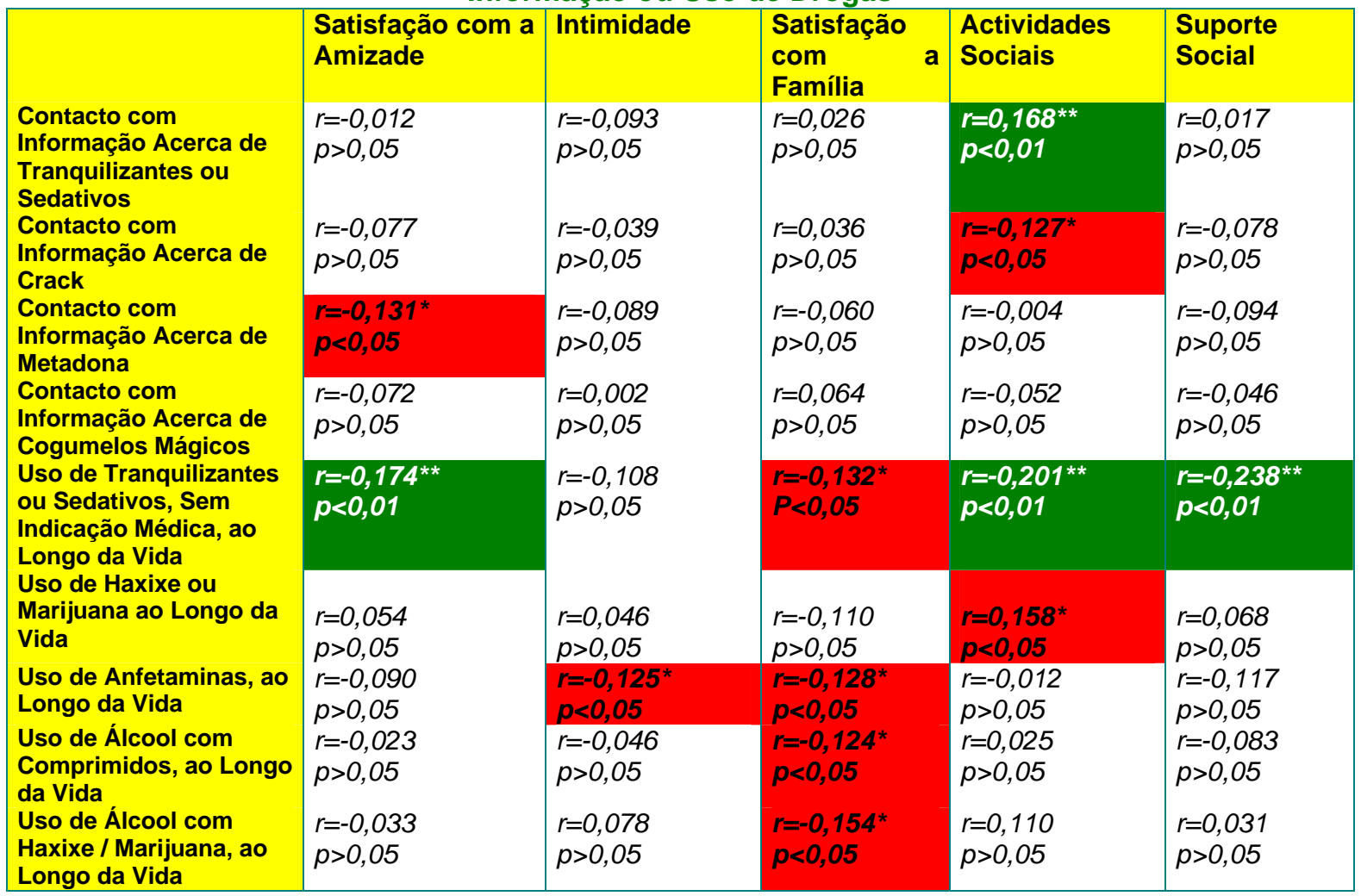

* Correlação estatisticamente significativa

** Correlação especialmente significativa 


\section{DISCUSSÃO}

Após a análise dos nossos resultados e comparativamente aos estudos anteriores, pode-se referir que:

No que diz respeito ao Consumo de Tabaco, os resultados do nosso estudo comparativamente com os obtidos pelo "ESPAD 99" e "ESPAD 03", apresentam percentagens mais elevadas.

Relativamente aos valores de Consumo de Tabaco ao Longo da Vida os resultados obtidos nos estudos efectuados vão sendo progressivamente mais elevados.

Quanto ao Consumo de Tabaco nos Últimos $\mathbf{3 0}$ dias, pode-se evidenciar a descida de valores do estudo "ESPAD 99" para o estudo "ESPAD 03", verificando-se, no nosso estudo, uma percentagem mais elevada.

Os resultados sugerem que o consumo de tabaco aumenta com a idade e com o nível de ensino verificando-se que a correlação entre as Variáveis Idade e Consumo de Tabaco nos Últimos 30 Dias é estatisticamente significativa.

No que diz respeito ao Consumo de Álcool, os resultados do nosso estudo, comparativamente com os obtidos pelo "ESPAD 99" e "ESPAD 03", apresentam, de uma forma geral, percentagens mais elevadas.

Em relação ao Consumo de Bebidas Alcoólicas nos Últimos 30 dia, ao Consumo de Cerveja e ao Consumo de Vinho, verifica-se uma ligeira diminuição de valores do estudo "ESPAD 99" para o "ESPAD 03". No nosso estudo os valores são mais elevados do que os encontrados nos estudos referidos.

É demonstrado, nos três estudos, um consumo preferencial de Bebidas Espirituosas/Destiladas, seguido do Consumo de Cerveja e por último do Consumo de Vinho. Tais factos sugerem que as bebidas mais consumidas são aquelas que se encontram mais em voga e as mais aceites actualmente no convívio social juvenil. Nos Bares e Pub's, referidos no nosso estudo como os locais preferenciais do último consumo. A oferta actual dirige-se essencialmente para bebidas desta natureza.

A cerveja surge como a segunda bebida mais consumida. Na nossa opinião deve-se ao facto de a cerveja ser uma bebida economicamente mais acessível, com maior publicidade em todos os tipos de mass media.

Os resultados, de uma forma geral, sugerem que o consumo de Bebidas Alcoólicas aumenta com a idade, com o nível de ensino e depende do sexo. Verificou-se que os indivíduos do sexo Masculino consumiram mais vezes Bebidas Alcoólicas e atingiram mais vezes estados de embriaguês (13,6\%). Contudo estes apresentam uma percentagem mais elevada $(5,9 \%)$ do que os indivíduos do sexo Feminino $(4,2 \%)$, em relação aos que nunca consumiram álcool. Tais factos podem dever-se ao Álcool surgir como um dos meios de integração social e ao facto de o álcool ser uma droga socialmente aceite.

No que diz respeito ao Consumo de Drogas, os resultados do nosso estudo comparativamente com os obtidos pelo "ESPAD 99" e "ESPAD 03", apresentam, igualmente, percentagens mais elevadas.

Relativamente ao Uso de Tranquilizantes ou Sedativos, sem Indicação Médica e ao Uso de LSD ou Outros Alucinogéneos, as percentagens obtidas têm sido progressivamente mais elevadas, nos 
sucessivos estudos.

Quanto ao Uso de Anfetaminas, verifica-se uma diminuição de valores do "ESPAD 99" para o "ESPAD 03", existindo um aumento dos mesmos no nosso estudo.

Em relação ao Uso de Crack, Cocaína e Ecstasy, verifica-se um aumento de valores do estudo "ESPAD 99" para o "ESPAD 03". No nosso estudo os valores são mais elevados do que os encontrados nos estudos referidos.

Pode-se constatar uma diminuição do Uso de Heroína nos três estudos, ao contrário do que se pode verificar no Uso dos “Cogumelos Mágicos”.

Quanto ao Uso de Esteróides Anabolizantes, não se verificou consumo com excepção do referido no estudo "ESPAD 03".

As drogas ilícitas mais consumidas ao longo da vida, por ordem decrescente, são os Tranquilizantes/Sedativos, sem Indicação Médica; "Cogumelos Mágicos”; Anfetaminas; LSD; Ecstasy; Cocaína; Heroína; Crack e Esteróides Anabolizantes.

Verifica-se um aumento no consumo da maioria das drogas ilícitas estudadas à excepção do consumo de Ecstasy, Cocaína, Heroína, Crack e dos Esteróides Anabolizantes, onde se verificou uma diminuição dos valores percentuais.

Na nossa opinião o consumo de drogas ilícitas passa um pouco por modas, que variam ao longo do tempo e com a realidade social. Derivado a este factor verifica-se uma diminuição do consumo de determinadas drogas (Ecstasy, Cocaína, Heroína, Crack e Esteróides Anabolizantes).

Por outro lado o consumo de drogas pode ser justificado por novos modos de utilização das drogas, por novas populações (os jovens) e por o fenómeno perder o seu estigma de gueto e ganhar uma dimensão ideológica e socialmente aceite. O consumo dos Tranquilizantes e Sedativos, o que apresenta um maior aumento no nosso estudo, pode sugerir uma forma de fuga da realidade do mundo e da depressão de cada um, uma resposta (falsa) alternativa para a angústia, para a rejeição, para a solidão, para a imaturidade, para a competitividade.

Apesar de se terem obtido valores percentuais elevados no que diz respeito ao contacto com informação acerca de drogas no nosso estudo, verifica-se simultaneamente elevados valores de consumo das mesmas. Esta situação sugere que as campanhas publicitárias/informativas contra o consumo de drogas não estão a surtir o efeito desejado.

Verificou-se no nosso estudo que a droga ilícita mais consumida é o Haxixe/Marijuana (35,9\%) o que se deve, na nossa opinião, a ser a droga ilícita mais disponível, mais barata e mais aceite nos grupos e na sociedade.

As variáveis relacionadas com o Consumo de Tabaco não apresentam correlação com o Suporte Social, contudo apresentam uma correlação estatisticamente significativa com a dimensão Satisfação com a Família. Como a correlação apresenta um valor negativo, podemos afirmar que quanto menor é a Satisfação com a Família, maior é o Consumo de Tabaco e vice-versa.

Tais resultados sugerem-nos que os conflitos/problemas familiares levam os adolescentes a consumirem tabaco como uma forma de resposta ao stress familiar ou até como forma de demonstração da 
sua afirmação por oposição às figuras parentais?

Também as variáveis correspondentes ao Consumo de Bebidas Alcoólicas, não apresentam correlação com o Suporte Social. Contudo apresentam uma correlação estatisticamente significativa com as dimensões Satisfação com a Família e Actividades Sociais. Como a correlação com a dimensão Satisfação com a Família apresenta um valor negativo, podemos afirmar que quanto menor é a Satisfação com a Família, maior é o Consumo de Bebidas Alcoólicas e vice-versa. A correlação com a dimensão Actividades Sociais apresenta valor positivo, o que significa que quantos maiores são as Actividades Sociais, maior é o Consumo de Bebidas Alcoólicas.

Tais resultados sugerem, à semelhança das variáveis relacionadas com o Consumo de Tabaco, que os conflitos/problemas familiares levam os adolescentes a consumirem Bebidas Alcoólicas como uma forma de resposta ao stress familiar e como forma de inserção social ao nível dos seus grupos de pares/amigos, pois também se encontra relacionado com as Actividades Sociais, de modo a compensarem o afastamento familiar. A participação e integração dos jovens em grupos está muitas vezes dependente da adopção de comportamentos comuns, de rituais de iniciação dos quais fazem, muitas vezes parte, os consumos de bebidas alcoólicas.

A única excepção nas variáveis correspondentes ao Consumo de Bebidas Alcoólicas, é a variável Consumo de Bebidas Alcoólicas durante os Últimos 30 Dias que apresenta uma correlação especialmente significativa com o Suporte Social, apresentando em simultâneo uma correlação especialmente significativa com as dimensões Actividades Sociais e Intimidade, e ainda uma correlação estatisticamente significativa com a dimensão Satisfação com a Amizade. Como todos os valores de correlação são positivos, pode-se dizer que quanto maior é a Satisfação com a Amizade, a Intimidade e as Actividades Sociais, maior é o Consumo de Bebidas Alcoólicas durante os Últimos 30 Dias.

Quanto ao Consumo de 5 ou mais Bebidas Alcoólicas de Seguida Durante os Últimos 30 Dias, esta variável apresenta uma correlação estatisticamente significativa com o Suporte Social, apresentando em simultâneo uma correlação especialmente significativa com a dimensão Actividades Sociais e estatisticamente significativa com as dimensões Satisfação com a Amizade e Intimidade. Como estes valores de correlação são positivos, podemos afirmar que quanto maior é a Satisfação com a Amizade, a Intimidade e as Actividades Sociais, maior é o Consumo de 5 ou mais Bebidas Alcoólicas de Seguida Durante os Últimos 30 Dias.

O resultado destas correlações sugerem-nos que o Consumo de Bebidas Alcoólicas durante os Últimos 30 Dias e o Consumo de 5 ou mais Bebidas Alcoólicas de Seguida Durante os Últimos 30 Dias, aumenta quando o adolescente se relaciona melhor com o grupo de amigos e quando frequenta mais eventos sociais.

No que respeita ao Consumo de Drogas ilícitas, mais propriamente no Uso de Tranquilizantes ou Sedativos, Sem Indicação Médica, ao Longo da Vida, esta apresenta uma correlação especialmente significativa com o Suporte Social. Ao mesmo tempo verifica-se uma correlação especialmente significativa com as dimensões Satisfação com a Amizade e Actividades Sociais, e ainda uma correlação estatisticamente significativa com a dimensão Satisfação com a Família. Como estes valores de correlação são negativos, podemos afirmar que quanto maior é a Satisfação com a Amizade, a Satisfação com a Família e as Actividades Sociais, menor o Uso de Tranquilizantes ou Sedativos, Sem Indicação Médica, ao 
Longo da Vida.

Isto sugere-nos que os adolescentes que têm conflitos/problemas familiares e com os amigos, sentem-se naturalmente mais deprimidos. Tais sentimentos e emoções podem conduzir à necessidade de consumir Tranquilizantes ou Sedativos. Assim a vontade de sair de casa e participar em actividades socais diminui também, devido á medicação e ao seu estado de espírito?

Quanto ao Uso de Haxixe ou Marijuana ao Longo da Vida, esta variável, não apresenta correlação com o Suporte Social, contudo verifica-se uma correlação estatisticamente significativa com a dimensão Actividades Sociais. Como o valor desta correlação é positivo, quanto maior são as Actividades Sociais, maior é o Uso de Haxixe ou Marijuana ao Longo da Vida.

Tais resultados sugerem-nos que o Consumo de Haxixe/Marijuana aumenta quando o adolescente frequenta mais eventos sociais, isto é onde esta droga se encontra, possivelmente, mais disponível e onde existe mais liberdade para a consumirem.

Relativamente ao Uso de Anfetaminas, ao Longo da Vida, esta variável, não apresenta correlação com o Suporte Social. Contudo verifica-se uma correlação estatisticamente significativa com as dimensões Intimidade e Satisfação com a Família. Como os valores de correlação são negativos podemos afirmar que quanto menor é a Intimidade e a Satisfação com a Amizade, maior é o Uso de Haxixe ou Marijuana ao Longo da Vida.

Tais resultados sugerem-nos que os adolescentes que possuem conflitos/problemas familiares consomem mais Anfetaminas como uma forma de resposta ao stress familiar e ainda quando se sentem menos satisfeitos com a suas relações com amigos íntimos.

Em relação ao Uso de Álcool com Comprimidos, ao Longo da Vida, esta variável, não apresenta correlação com o Suporte Social, contudo verifica-se uma correlação estatisticamente significativa com a dimensão Satisfação com a Família. Como o valor de correlação é negativo, podemos afirmar que quanto menor é a Satisfação com a Família, maior é o Uso de Álcool com Comprimidos, ao Longo da Vida.

Por último, no que diz respeito ao Uso de Álcool com Haxixe / Marijuana, ao Longo da Vida, não apresenta correlação com o Suporte Social, contudo verifica-se uma correlação estatisticamente significativa com a dimensão Satisfação com a Família. Como o valor de correlação é negativo, refere-se que quanto menor é a Satisfação com a Família, maior é o Uso de Álcool com Haxixe / Marijuana, ao Longo da Vida.

Na nossa opinião, o adolescente consome mais Álcool com Comprimidos e Álcool com Haxixe / Marijuana como uma forma de resposta ao stress gerado pelos problemas/conflitos familiar. Pensamos que os adolescentes combinam estas substâncias devido a serem as 3 mais consumidas no nosso estudo. Apesar da correlação destas duas variáveis com a dimensão Actividades Sociais não terem um valor de correlação estatisticamente significativo, as variáveis (Consumo de Álcool ao Longo da Vida, Uso de Haxixe ou Marijuana ao Longo da Vida e Uso de Tranquilizantes ou Sedativos, Sem Indicação Médica, ao Longo da Vida) apresentam correlação com a dimensão Actividades Sociais. 


\section{REFERÊNCIAS BIBLIOGRÁFICAS}

RIBEIRO, J.L.P. - Escala de Satisfação com o Suporte Social. Análise Psicológica. 1999, 3 (XVII) pág. 547558.

\section{Agradecimentos}

Escola Superior de Enfermagem de Portalegre 